\title{
Prevalence and Factors Associated with Stunting among Public Primary School Pupils in Kasulu District, Western Tanzania.
}

\author{
Jairos N. Hiliza ${ }^{a^{*}}$, Leyna Germana ${ }^{b}$, Amalberga Kasangalac, Flora Joram ${ }^{d}$ \\ 'Department of Epidemiology and Biostatistics Muhimbili University of Health and Allied Sciences, 'bTanzania Field Epidemiology and Laboratory \\ Training Programme, cMinistry of Health, Community Development, Gender, Elderly and Children, dDepartment of Community Development Studies \\ Muhimbili University of Health and Allied Sciences \\ Correspondence to Dr. Jairos N Hiliza (nsengiyumva1987@gmail.com)
}

\begin{abstract}
Background: Underfeeding of a child in the first 2 years of life results in irreversible growth damage. Globally, stunting has declined from 39.7\% in 1990 to $26.7 \%$ in 2010 while in Africa has remained at $40 \%$ since 1990 . However, stunting is little known in primary pupils.

This study estimated the prevalence of stunting and contributing factors among public primary school pupils in Kasulu District.

Method: Cross-sectional study was conducted among public primary pupils. Systematic random sampling was used to select study participants and then stratified to 5-7 and 8-12 years. Socio-economic factors, dietary practices, water, sanitation, and hygiene behaviours; school performance/attendance data were collected using a pretested questionnaire. Measurements were standardised to the World Health Organization HAZ-Scores for both girls and boys. Descriptive statistics, bivariate, and multivariable logistic regression were used to generate results.

Results: A total of 400 pupils (100\%RR) were recruited into the study, mean age of 7.51 (STD=1.54) years and a half $(50.3 \%)$ were boys. The prevalence of stunting was $127(31.8 \%)(95 \% \mathrm{Cl}: 27.2 \%-36.6 \%)$, with no sex difference (63 $(31.7 \%)$ - girls vs. $64(31.8 \%)$ - boys; $p=0.969)$. Household wealth influenced stunting; lowest quintile (AOR=28; $95 \% \mathrm{Cl}: 3.64-214.6 ; \mathrm{p}<0.001$ ) 2nd quintile (AOR = 17; 95\%Cl: $2.20-138.5 ; \mathrm{p}<0.01$ ), the 3rd quintile (AOR $=8.0 ; 95 \% \mathrm{Cl}: 0.99-64.67 ; \mathrm{p}=0.051)$ and 4 th quintile $(\mathrm{AOR}=4.2 ; 95 \% \mathrm{Cl}: 0.49-36.75 ; \mathrm{p}=0.191)$ when compared to 5th (highest) wealthquintile. Food insecurity $(A O R=10.6 ; 95 \% \mathrm{Cl}: 4.60-24.60 ; \mathrm{p}<0.001)$, less protein in meal were the risk for stunting $(A O R=14.6 ; 95 \% \mathrm{Cl}: 4.07-52.42 ; \mathrm{p}<0.001)$. Inappropriate hand wash after toilets both at school, (AOR=3.5; 95\%Cl: 1.62-7.58; $p=0.001)$, and home (AOR = 13.0; $95 \% \mathrm{Cl}: 2.73-61.76 ; \mathrm{p}=$ 0.001 ) were the risk for stunting. Stunted pupils had irregular school attendance $(A O R=9.4 ; 95 \% \mathrm{Cl}: 4.42-19.93$; $\mathrm{p}<0.001)$ and poor performance $(A O R=23.6 ; 95 \% \mathrm{Cl}: 10.24-54.19 ; \mathrm{p}<0.001)$. Food insecurity influenced poor performance $(A O R=3.9 ; 95 \% \mathrm{Cl}: 1.67-8.92 ; \mathrm{p}<0.01)$ and irregular school attendance $(\mathrm{AOR}=5.4, \mathrm{p}=0.000)$.

Conclusion: Stunting among public primary school pupils is very high despite the prevention effort. Low wealth, food insecurity, poor hand hygiene, and lack of protein in a meal significantly influence stunting. Also, it affects the pupils' academic performance and attendance, availability of food in both quantity and quality, community nutrition
\end{abstract}

\section{INTRODUCTION}

$\triangle$ dequate nutrition during infancy and early A childhood is a fundamental prerequisite of each child's full human developmental potential. Thus, the period from conception through birth to 2 years of age is a vital window for child optimal growth, health, and behavioural development. ${ }^{2}$ Literature shows that, undernourishment during the first 1000 days, from pregnancy through the child's second birthday, results to long term and irreversible growth damage, with impacts observed at the individual, community, and nation as whole. ${ }^{3}$ In the joint United Nations International Children's Emergency Fund (UNICEF), World Health Organization (WHO) and World Bank 2013 report, 162 million children under the age of five are affected by stunting globally either during pregnancy or after delivery; this being only a twopercentage-point lower than it was 5 years ago. ${ }^{4,5}$ If the current trend is to continue, projections in East Africa show that $42.1 \%$ of children under five years of age will be stunted in $2025 .{ }^{6}$ Studies have indicated that stunting can be inherited from one generation to the next. ${ }^{7,8}$ This may occur when an undernourished woman bears low birth weight babies, who if are subjected to suboptimal feeding practices and high rate of infectious diseases, do not experience the catch-up growth in subsequent years leading to an intergenerational cycle of stunting. ${ }^{7}$ United Nations International Children's Emergency Fund (UNICEF) describes four forms in which under nutrition can m- 
anifest: underweight (<-1SD weight-for-age Z-score), wasting (<1SDweight-for-height), and stunting (<-1SD height-for-age). In 2010 UNICEF reported among these 3 forms of under nutrition, stunting was globally higher - for the year 2015 the global prevalence of stunting was $42 \%$ in comparison to wasting and underweight which were $5 \%$ and $16 \%$, respectively. ${ }^{2}$ A similar trend was observed in the Joint UNICEF, WHO and World Bank report of 2012 whereby $26 \%$ of the global under-fives population were stunted, $8 \%$ wasted and $11 \%$ underweight. ${ }^{8}$ The stunting was reported to be caused by the chronic lack of adequate nutritious food, poor child care practices, lack of access to health, and other social services. ${ }^{8}$

Forty-two percent $(42 \%)$ of children are affected by stunting either during pregnancy or after delivery globally. The prevalence of stunting in Tanzania is estimated to be $35 \% .^{3}$ Kigoma region is one of the regions with a "very high" prevalence of stunting in children less than five years, estimated at $35 \% .^{2}$

Bad feeding habits, number of household members, frequent infectious diseases attack, unhealthy environment, inadequate care practices, poverty, illiteracy, socio norms and Water, Sanitation, and Hygiene practices are determinants of stunting commonly described in most literature on stunting among under five years of age. We assumed that the distribution of these determinants may be different among school children as they were not under total parental care and can secure food on their own if available. They also may access food at school as well as other health interventions such as de-worming and schistosomiasis treatment. To ascertain whether these determinants affect primary school children in the same way as they do among under-five children, we conducted this study aiming at estimating the prevalence of stunting and its associated factors among primary school children in Kasulu District.

\section{MATERIALS AND METHODS}

\section{Study Area and Design.}

This was a cross-sectional study conducted in Kasulu District involving primary school pupils attending public schools by January 2018. Kasulu District Council was purposefully selected among the eight councils in Kigoma region, due to its high population density. Food adequacy in the district is determined by cross broader trade across the national border and/or inter-regional boundaries which have tended to shift large quantities of food across the border to neighbouring regions or countries. The most cultivated crops are maize, cassava, potatoes, and beans. ${ }^{31}$ Kasulu district has been the recipient of the highest number of refugees and asylum seekers from the Republic of Burundi and the Democratic Republic of Congo. The total district population was 556,851; $273,904(49.2 \%)$ being male, $50.7 \%$ were children aged between 5-12 years as projected by the National Bureau of Statistics in the 2018 dashboard. The population growth was estimated at 2.4, primary school enrolment Was about $59 \%$, and an illiteracy rate of $23 \% .{ }^{32}$ Kasulu district is highly populated in the region contributing to $20 \%$ of the region population with an average household size of $7.6 .^{9}$ It also hosts more refugees than any other district in the region. The district has a total of 79 public primary schools with no private primary schools, 38 disp- ensaries, and 5 health centres.

\section{Sample Size Calculation}

The sample size was calculated using Leslie's formula. The estimation of the sample size based on the stunting prevalence of $38 \%$ taken from a study done by Semali et $a l^{14}$ in Kongwa District Tanzania, a site with similar settings as ours, with a marginal error of $5 \%$ and $95 \%$ confidence level. A response rate of $90 \%$ was used to adjust the estimated sample size resulting in a minimum sample size of 400 study participants.

The sample size (n) was calculated using Leslie's formula

$$
\begin{gathered}
n=Z^{2} \times P(\underline{100-P)} \\
\varepsilon^{2} \\
\text { Where }(n)=\text { Sample size }
\end{gathered}
$$

$Z=1.96$ (95\% confidence interval) in two tailed t-test

$$
\begin{gathered}
\mathrm{P}=\text { Prevalence of stunting of } 38 \% .^{14} \\
\varepsilon=\text { Marginal error } 5 \% \\
\mathrm{n}=1.96^{2} \times 38 \% \times \frac{(100-38 \%)}{52}=362 \sim 360
\end{gathered}
$$

Expected response rate $(\mathrm{RR})=90 \%$

Adjusting for non-response, then,

$$
\begin{gathered}
\mathrm{n}=360 / 90 \% \\
=400
\end{gathered}
$$

Therefore, the minimum study sample size was 400 participants

\section{Sampling Technique}

We used a systematic sampling technique to select three wards and one village from the respective ward. Kitema village, Kibirizi village, and Bugaga village were randomly selected from Nyenge Ward, Buhoro Ward, and Bugaga Ward respectively, and one public primary school from each village was randomly selected. Proportion probability sampling was used to estimate the sample size for each public primary school selected. The study respondents were stratified into two age groups: 5 to 7 years whose parents/guardians were traced at home to complete responses of the household questionnaire as their responses to some questions were not reliable; and 8 to 12 years who self-administered the questionnaire.

(Figure 1)

The $\mathrm{k}^{\text {th }}$ interval depended on the number of eligible study participants in each school and each age strata. The formula below was used to calculate the kth interval

$$
\mathrm{k}=\mathrm{N} / \mathrm{n}
$$

Where;

$\mathrm{k}$ is a sampling interval (sometimes called a skip) $\mathrm{N}$ is the number of eligible study population size $\mathrm{n}$ is the required sample size 


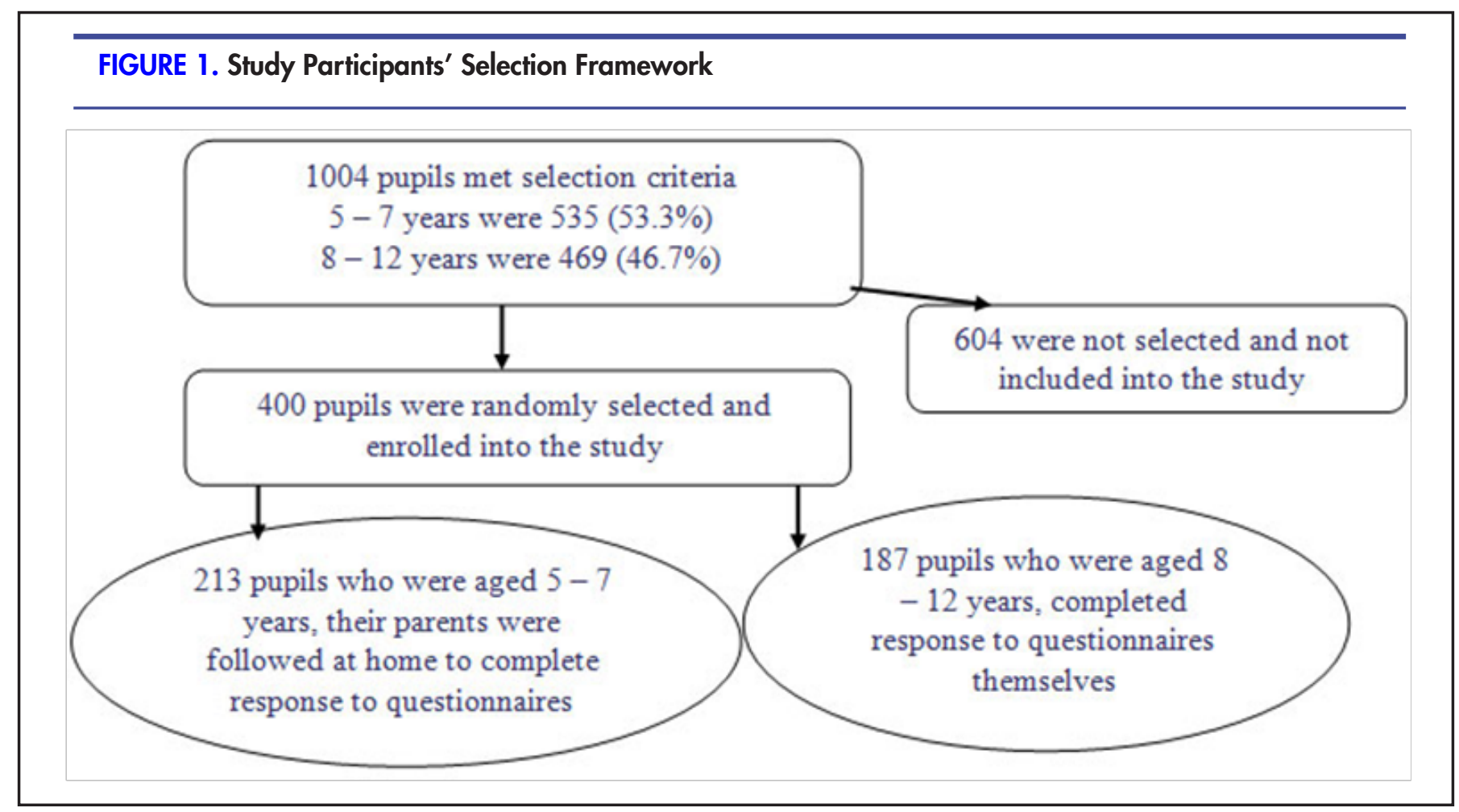

FIGURE 2. Prevalence of Stunting by Sex in Public Primary School Pupils in Kasulu District

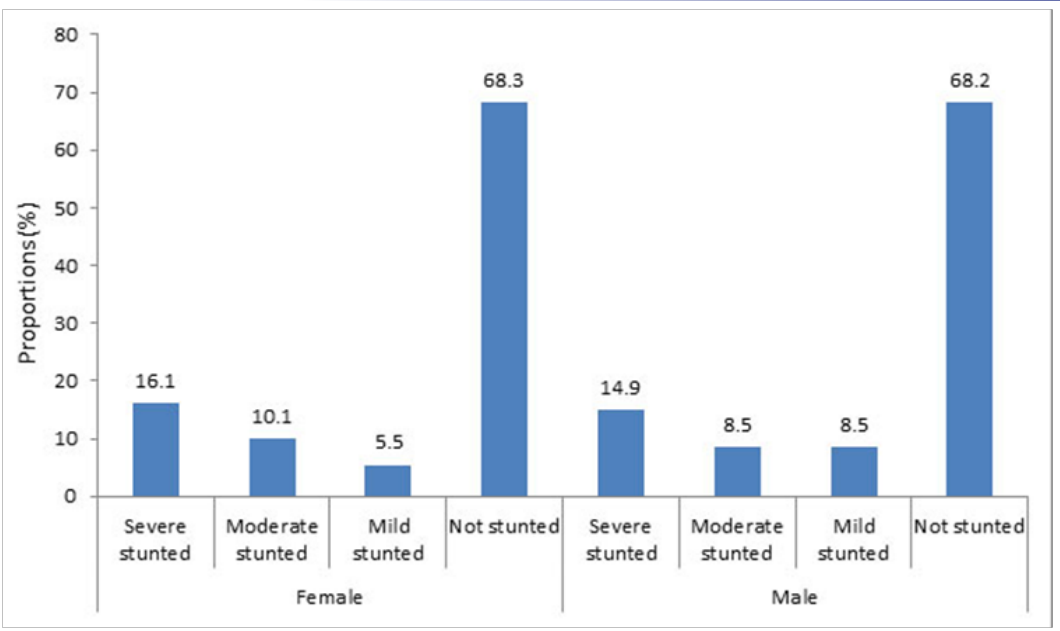

Data Collection Instruments and Procedures

A questionnaire was developed in English then translated to Swahili and back-translated to English to ensure a correct translation of meaning. Pre-testing of questionnaires was done to test for clarity of questions, validity, reliability, feasibility, and study logistics. This also assisted the research assistants to exercise flexibility in the wording of questions contained in a questionnaire. The pre-testing results were used to modify the content and wording of the questionnaire. The questionnaire had both closed and open-ended questions. The closeended questions provided more structured responses to aid precision of responses to standardize analysis. The open-ended questions provided additional information that may not have been captured in the close-ended questions. The type of data collected included: sociodemographic and economic data, dietary practices, sanitation practices, school performance, and school attendance. The questionnaires were administered by a 
Principle researcher and two trained research assistants.

\section{Anthropometric Measurements}

We measured height in centimetres to the nearest $0.1 \mathrm{~cm}$. The respondent stood upright on a uniformly calibrated height board facing forward without shoes. Height-forage Z scores were calculated for boys and girls separately. WHO 2006 references were used to define stunting $\mathrm{HAZ}<-1 \mathrm{SD}$ was used to create the outcome variables.

\section{Data Management and Analysis}

The responses to open-end questions were grouped then coded to respective codes in a questionnaire. The complete filled and coded questionnaires were entered in the Epi Info ${ }^{\mathrm{TM}}$ make view; a trademark of the Centre for Diseases and Control, and then transferred to STATA software version 13 owned by StataCorp LP, 4905 Lake way Drive, College Station, Texas 77845, United States of America, for analysis. After data cleaning, frequencies, and percentages of independent variables were summarized in tables. Univariate, bivariate, and multivariable logistic regression models (inclusion criteria were any variable with p-value $<0.2$ ) were built to examine demographic characteristics and their association with stunting. We created population wealth quintiles by Principal Component Analysis. We reported Odd Ratios and their $95 \%$ Confidence Interval, and the significance level of alpha $<5 \%$.

\section{Ethical Considerations}

We obtained ethical clearance to conduct the study from the Muhimbili University of Health and Allied Sciences Institutional Review Board. We sought permission to conduct this study from the Kasulu district authority. The head teacher signed written informed consent and parent/ guardian consented on behalf of children. We explained study objectives, methodology, and benefits to the head teachers of respective primary school and parent/guardian of each study participant. Study participants aged 8 years and older assented before recruitment into the study. All participants were free to withdraw from the study at any point in time. We assigned numbers to participants to assure confidentiality and only the researcher and research team accessed the respondent questionnaires

\section{RESULTS}

\section{Demographic Characteristics of the Study Population}

We enrolled a total of 400 pupils with a mean age of 7.51 $(\mathrm{SD}=1.54)$ years with a $100 \%$ respondents response rate to the study. More than half $213(53.2 \%)$ were aged between 5 to 7 years, and about half $201(50.3 \%)$ were boys. More than half of the respondents 201 (50.3\%) were in class two, while $123(30.8 \%)$ were in class one and $76(19.0 \%)$ were in class three. About forty percent of $159(39.8 \%)$ of the pupils were from Kibirizi primary school. The mean age of the parents/guardians was 32.4 ( $\mathrm{SD}=5.09)$ years, with a majority of $243(60.8 \%)$ aged between 26-35 years. More than half of the parents/ guardians $211(52.3 \%)$ had primary education, while only $50(12.4 \%)$ had secondary education or above. The majority of $248(62 \%)$ of the parents/guardians were either peasants or livestock keepers or about 1 in 5 were involved in petty business $86(21.5 \%), 57(14.3 \%)$ were skilled labourers and only $9(2.2 \%)$ were employed.
Almost all parents/guardians were married or cohabiting $366(91.5 \%)$ (Table 1$)$

\section{Prevalence of Stunting Among Study Respondents}

The overall prevalence of stunting was 127 (31.8\%) (95\% CI: $27.2 \%-36.6 \%$ ), where $63(31.7 \%)$ girls and 64 $(31.8 \%)$ boys were considered as stunted. The distribution of stunting among girl's pupils was $5.5 \%$ mild, $10.1 \%$ moderate, and $16.1 \%$ severely stunted; and boys were $8.5 \%$ mild, $8.5 \%$ moderate, and $14.9 \%$ stunted. (Figure 2).

\section{Socio-Economic Determinants of Stunting}

Older pupils 8 -12years of age were significantly more likely to be stunted $70(37.4 \%)$ as compared to younger pupils $5-7$ years 57 (26.8\%; $p=0.022)$. Stunting was not differentially distributed by sex (boys $64(31.8 \%)$ and girls $63(31.7 \% ; p=0.969)$. Pupils in lower classes one $41(33.3 \%)$ and two70 (34.8) were more stunted than pupils in class three $16(21.1 \%$; $\mathrm{p}=0.081)$.

Older parents/guardians ( $>35$ years) had a higher probability of $76(60.3 \%)$ of having a child who was stunted than parents/guardians aged 26-35 years 44 $(18.1 \%)$ or parents/guardians age $<26$ years 7 (22.6\%; $\mathrm{p}<0.001)$. Having no formal education had a higher likelihood of $62(44.6 \%)$ of having a stunted child than primary education $56(26.5 \%)$ and secondary education or higher $9(18.0 \% ; \mathrm{p}<0.001)$. Being either a peasant or livestock keeper was associated with a significantly higher probability of having a stunted child 91 (37.5\%) compared to those who were employed $2(22.2 \%)$, skilled labourers $9(15.8 \%)$ or in petty business $25(28.4 \%$; $p=$ $0.014)$.

Parents/guardians who were categorised as not being in marriage (single, separated, divorced, or widow) had a significantly higher likelihood of having a stunted child than those who were married or cohabiting 24 (70.6\%) vs. $103(28.1 \% ; \mathrm{p}<0.001)$. A household, which had six or more children, were significantly more likely to have a stunted child $73(59.8 \%)$ than households with five or less $54(19.4 \%$; $<<0.001)$. Also, pupils not living with both of their parents (categorized as others) had a higher proportion of stunting compared to those who reported living with both parents $31(68.9 \%$ vs. 96 (27.0\%; $\mathrm{p}<0.001)$. Households ranked as having lower economic status were significantly associated with stunting than households ranked in the highest economic status where $3(3.8 \%), 11(14.1 \%), 18(22.0 \%), 42(53.2 \%)$, $53(65.4 \%)$ percent of the children were categorised as stunted from lowest, low, middle, high and highest quintile, respectively $(\mathrm{p}$ trend $<0.001$ )

\section{The Influence of Dietary Practices on Stunting}

Pupils reporting coming from households with inadequate food were more likely to be stunted compared to pupils coming from households reported to have adequate food supply $97(84.4 \%)$ vs. 30 (10.5\%; p<0.001). Reporting eating less than 2 meals a day had a $108(61.7 \%)$ probability of stunting compared to $19(8.4 \%)$ in pupils reporting eating 3 meals $(p<0.001)$. Stunting was not differentially distributed by food diversity (starch only 65 $(32.3 \%)$, starch and protein $23(26.1 \%)$, starch protein and fats $6(33.3 \%)$ and starch, protein, fats and vegetables 
TABLE 1: Demographic Characteristics of Pupils and their Parents/Guardians

\begin{tabular}{|c|c|c|c|c|c|}
\hline \multicolumn{3}{|c|}{ Pupils characteristics } & \multicolumn{3}{|c|}{ Parent/guardian characteristics } \\
\hline Variable & $\mathbf{N}$ & $\%$ & Variable & $\mathbf{N}$ & $\%$ \\
\hline \multicolumn{3}{|l|}{ Age, Years } & \multicolumn{3}{|l|}{ Age, Years } \\
\hline Mean (SD 1) & 7.5 & 1.54 & Mean (SD) & 32.4 & 5.09 \\
\hline $5-7$ & 213 & 53.3 & $<26$ & 31 & 7.7 \\
\hline $8-12$ & 187 & 46.8 & $26-35$ & 243 & 60.8 \\
\hline \multicolumn{3}{|l|}{ Sex } & & 126 & 31.5 \\
\hline Girls & 199 & 49.8 & \multicolumn{3}{|l|}{ Marital status } \\
\hline Boys & 201 & 50.3 & Not married* & 34 & 8.5 \\
\hline \multicolumn{3}{|l|}{ Class } & Married/cohabiting & 366 & 91.5 \\
\hline One & 123 & 30.8 & \multicolumn{3}{|l|}{ Education } \\
\hline Two & 201 & 50.3 & No formal education & 139 & 34.8 \\
\hline Three & 76 & 19 & Primary education & 211 & 52.8 \\
\hline \multicolumn{3}{|l|}{ School } & Secondary and above & 50 & 12.4 \\
\hline Kitema & 125 & 31.2 & \multicolumn{3}{|l|}{ Occupation } \\
\hline Kibirizi & 159 & 39.8 & $\begin{array}{l}\text { Peasant/livestock } \\
\text { keepers }\end{array}$ & 248 & 62 \\
\hline \multirow[t]{3}{*}{ Bugaga } & 116 & 29.0 & Petty business & 86 & 21.5 \\
\hline & & & Skilled labourers & 57 & 14.3 \\
\hline & & & Employed & 9 & 2.2 \\
\hline
\end{tabular}

/fruits 33 (35.5\%; $p=.587)$. Pupils reported eating proteins' food less frequently had a higher chance of being stunted than children who reported eating proteins' food frequently $(p<.001)$. There was no difference observed between sharing $103(31.4 \%)$ and not sharing plate $24(33.3 \%)$ during eating $(p=.750)$.

\section{The Influence of Hand Hygiene Practices to Stunting}

Not washing hands after visiting the toilet at school increased the chance of stunting by 103 (52.3\%) in comparison with washing hands with running water only or with soap which was $24(11.8 \%$; p $<0.001)$. Similarly, not washing hands at home after visiting the toilet was associated with a $39(92.9 \%)$ increased probability of stunting compared to washing hands with running water only or with soap $88(24.6 \% ; p<.001)$

\section{Multivariable Analysis of Stunting By Social-Economic, Dietary, Hygiene, and Sanitation Factors}

Socio-demographic characteristics that were significantly associated with stunting in this population were household wealth quintiles, household food security, and frequency of eating proteinous food and hand wash practices at both school and home. Household wealth had a linear relationship with stunting where pupils from the lowest quintile had a 28 fold increase chance of being stunted $(\mathrm{AOR}=28 ; 95 \%$ CI: $3.64-214.6 ; \mathrm{p}<0.001)$ followed by the 2 nd quintile $(\mathrm{AOR}=17 ; 95 \% \mathrm{CI}: 2.20-138.5 ; \mathrm{p}<0.01)$, the 3rd quintile (AOR $=8.0 ; 95 \%$ CI: $0.99-64.67 ; p=.051)$ and 4 th quintile $(\mathrm{AOR}=4.2 ; 95 \% \mathrm{CI}: 0.49-6.75 ; p=.191)$ when compared to 5 th (highest) wealth quintile.
Pupils who reported inadequate food in the household had 11 times more likelihood of being stunted than pupils who reported adequate food in their households (AOR $=10.6 ; 95 \%$ CI: $4.60-24.60 ; \mathrm{p}<0.001)$. The probability of being stunted increased by a factor of 14 times if the eating of proteinous food occurred once a month or less compared to those who ate proteinous foods 3 times per week $(\mathrm{AOR}=14.6 ; 95 \% \mathrm{CI}: 4.07-52.42 ; p<.001)$.

Improper hands wash after toilet both at school and the home was significantly associated with stunting. Those who reported not to wash hands after toilets at school were at higher risk of being stunted $(\mathrm{AOR}=3.5 ; 95 \% \mathrm{CI}$ : $1.62-7.58 ; p=.001)$, compared to those who used either water only or with soap for washing hands after toilets. At home pupils who reported not to wash hands were at 13 folds increase at risk of being stunted (AOR $=13.0$; 95\% CI: $2.73-61.76 ; p=0.001$ )

Relationship between Stunting and School Attendance and Academic Performance

Pupils who were stunted had a higher proportion of irregular school attendance $35(27.6 \%)$ compared to pupils who were not stunted $10(3.7 \% ; p<.000)$. Being stunted $68(77.3 \%)$ was associated with a significantly higher chance of failing at the end of year examination compared to those who were not stunted $13(6.7 \%$; $p<0.001)$.

Multivariable Analysis of School Attendance and Academic Performance by Stunting 


\begin{tabular}{|c|c|c|}
\hline Variables & Crude OR $\left(95 \% \mathrm{Cl}^{\mathrm{a}}\right)$ & Adjusted OR $\left(95 \% \mathrm{Cl}^{b}\right)$ \\
\hline \multicolumn{3}{|l|}{ Age, in years } \\
\hline $\begin{array}{l}5-7 \\
8-12\end{array}$ & Ref & \\
\hline \\
\hline $\begin{array}{l}\text { Sex } \\
\text { Girls }\end{array}$ & & \\
\hline $\begin{array}{l}\text { Girls } \\
\text { Boys }\end{array}$ & $\begin{array}{l}\text { Ref } \\
1.0(0.66-1.54)\end{array}$ & NA \\
\hline \multicolumn{3}{|l|}{ Class of the pupils } \\
\hline One & *1.9 (0.96-3.65) & NA \\
\hline Two & $* * 2(1.07-3.74)$ & NA \\
\hline Three & Ref & \\
\hline \multicolumn{3}{|l|}{ Parent/guardian characteristics } \\
\hline$<26$ & $1.3(0.53-3.25)$ & NA \\
\hline $26-35$ & Ref & \\
\hline \multirow{2}{*}{\multicolumn{3}{|c|}{ Parent/quardian education }} \\
\hline & & \\
\hline No formal education & $3.7(1.66-8.12)^{* * *}$ & NS \\
\hline Primary education & $1.6(0.75-3.60)$ & NS \\
\hline \multirow{2}{*}{\multicolumn{3}{|c|}{ Parent/guardian Occupation }} \\
\hline & & \\
\hline Peasants/Livestock keepers & $3.1(1.47-6.68)^{* *}$ & NS \\
\hline Petty business & $2.1(0.91-4.95)$ & NS \\
\hline Skilled labour & Ref & \\
\hline Employment & $1.5(0.27-8.55)$ & NS \\
\hline \multicolumn{3}{|l|}{ Marital status } \\
\hline Not married & $6.1(2.83-13.26)^{* * *}$ & NS \\
\hline \multirow{2}{*}{\multicolumn{3}{|c|}{ Number of children in household }} \\
\hline & & \\
\hline 5 or less & Ref & \\
\hline 6 or more . & $* * * 6.2(3.87-9.87)$ & NS \\
\hline \multicolumn{3}{|l|}{ A person living with a child } \\
\hline $\begin{array}{l}\text { Both parents } \\
\text { Others }\end{array}$ & $\begin{array}{l}\text { Ref } \\
6.0(3.05-11.711)^{* * *}\end{array}$ & NS \\
\hline \multicolumn{3}{|l|}{ Household wealth quintiles } \\
\hline Lowest & $48.6(14.05-168.05)^{* * *}$ & $28.0(3.64-214.6)^{* * *}$ \\
\hline Low & $29.1(8.47-100.10)^{* * *}$ & $17.4(2.20-138.5)^{* *}$ \\
\hline Medium & $7.2(2.03-25.61)^{* *}$ & $8.0(0.99-64.67)$ \\
\hline High & $4.2(1.12-15.74)^{* *}$ & $4.2(0.49-36.75)$ \\
\hline Highest & Ref & Ref \\
\hline \multicolumn{3}{|l|}{ Household food security } \\
\hline Adequate & Ref & Ref \\
\hline Inadequate & $45.8(24.41-85.95)^{* * *}$ & $10.6(4.60-24.60)^{* * *}$ \\
\hline \multicolumn{3}{|l|}{ Number of meals per 24 hours } \\
\hline Two or less & $17.5(9.98-30.60)^{* * *}$ & NS \\
\hline \multirow{2}{*}{\multicolumn{3}{|c|}{ Frequency of eating protenous food }} \\
\hline & & \\
\hline Three times per week & Ref & Ref \\
\hline Once per week & $1.4(0.63-3.14)$ & $1.1(0.37-3.30)$ \\
\hline More than once per month & $21.3(10.90-41.74)^{* * *}$ & $3.0(1.14-.17)^{* * *}$ \\
\hline Once or less than once per month & $59.2(22.60-155.0)^{* * *}$ & $14.6(4.7-54.42)^{* * *}$ \\
\hline \multicolumn{2}{|l|}{ Hand wash practices after visiting toilet at school } & $35(162-758) * * *$ \\
\hline $\begin{array}{l}\text { Do not wash hands } \\
\text { Wash with water only or with soap }\end{array}$ & $\begin{array}{l}8.2(4.91-13.60)^{* 3 \times} \\
\text { Ref }\end{array}$ & $\begin{array}{c}3.5(1.62-7.58) \times 3 \times \\
\text { Ref }\end{array}$ \\
\hline \multicolumn{3}{|l|}{ Hand wash practices after visiting toilet at home } \\
\hline Do not wash hands & $39.9(12.03-132.0)^{* * *}$ & $13.0(2.73-6176)^{* * *}$ \\
\hline Wash with water only or with soap & Ref & Ref \\
\hline
\end{tabular}


TABLE 3: Logistic Regression Analysis Academic Performance and School Attendance by Stunting Status and Socio-Demographic Factors

\begin{tabular}{|c|c|c|c|c|}
\hline & $\begin{array}{l}\text { School attendance } \\
\text { Crude OR } \\
(95 \% \mathrm{Cl})\end{array}$ & $\begin{array}{l}\text { Adjusted OR } \\
(95 \% \mathrm{Cl})\end{array}$ & $\begin{array}{l}\text { Academic performance } \\
\text { Crude OR } \\
(95 \% \mathrm{Cl})\end{array}$ & $\begin{array}{l}\text { Adjusted OR } \\
(95 \% \mathrm{Cl})\end{array}$ \\
\hline \multicolumn{5}{|l|}{ Stunting status } \\
\hline Not stunted & Ref & Ref & Ref & Ref \\
\hline Stunted & $\begin{array}{c}10 \\
(4.77-21.0)^{* * *}\end{array}$ & $\begin{array}{c}9.4 \\
(4.42-19.93)^{* * *}\end{array}$ & $\begin{array}{c}47.3 \\
(22.3-100)^{* * * *}\end{array}$ & $\begin{array}{l}23.6 \\
(10.24-54.19) * * *\end{array}$ \\
\hline \multicolumn{5}{|l|}{ Household food security } \\
\hline Adequate & Ref & & Ref & Ref \\
\hline Inadequate & $\frac{6.3}{(3.26-12.37)^{* * *}}$ & NS & $\begin{array}{c}17.8 \\
(9.37-33.7)^{* * *}\end{array}$ & $\begin{array}{l}3.9 \\
(1.67-8.92)^{* *}\end{array}$ \\
\hline \multicolumn{5}{|l|}{ Pupils age group in years } \\
\hline $5-7$ & Ref & & & \\
\hline $8-12$ & $\begin{array}{l}4.1 \\
(2.0-8.31)^{* * *}\end{array}$ & $\begin{array}{c}3.7 \\
(1.73-7.73) * * *\end{array}$ & NA & \\
\hline \multicolumn{5}{|c|}{ Number of meals per 24 hours } \\
\hline Two or less & 5.4 & & 8.5 & \\
\hline Three & $(2.58-11.20)^{* * *}$ & NS & $(4.56-15.83)^{* * *}$ & NS \\
\hline \multicolumn{5}{|l|}{ Pupil's class } \\
\hline One & $\begin{array}{l}\text { Ref } \\
4.5\end{array}$ & & & \\
\hline Two & $\begin{array}{l}(1.69-11.8)^{* *} \\
2.8\end{array}$ & NS & NA & NA \\
\hline Three & $(0.87-8.83)$ & NS & NA & NA \\
\hline \multicolumn{5}{|c|}{ Frequency of eating protenous food } \\
\hline Three times per week & Ref & & Ref & \\
\hline Once a week & 1.3 & & 1.3 & \\
\hline$>$ Once per month & $\begin{array}{l}(0.48-3.52) \\
3.0\end{array}$ & NS & $\begin{array}{c}(0.53-3.23) \\
9.1\end{array}$ & NS \\
\hline 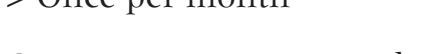 & $(1.32-6.62)^{* *}$ & NS & $(4.41-18.84)^{* * *}$ & NS \\
\hline Once or < once per month & $\begin{array}{l}* * * 4.6 \\
(1.98-10.8)\end{array}$ & NS & $\begin{array}{c}24.2 \\
(9.12-64.19)^{* * *}\end{array}$ & NS \\
\hline \multicolumn{5}{|l|}{ A person living with a child } \\
\hline Both parents & & & $\begin{array}{l}\text { Ref } \\
2.8\end{array}$ & \\
\hline Others & NA & NA & $(1.35-6.02)^{*}$ & NS \\
\hline \multicolumn{5}{|l|}{ Household wealth quintiles } \\
\hline Lowest & $\begin{array}{l}4.0 \\
(1.25-12.65)^{*}\end{array}$ & NS & $\begin{array}{c}16.3 \\
(4.61-57.84)^{* * * *}\end{array}$ & NS \\
\hline Low & & & 14.4 & \\
\hline Medium & $(1.41-14.09)^{*}$ & NS & $(4.04-51.0)^{* * *}$ & NS \\
\hline Miedrum & $\begin{array}{c}2.6 \\
(0.79-8.79)\end{array}$ & NS & $\begin{array}{c}4.8 \\
(1.29-18.06)^{* * *}\end{array}$ & NS \\
\hline High & & & 1.5 & \\
\hline & $(0.89-2.81)$ & NS & $(0.32-7.17)$ & NS \\
\hline Highest & Ref & & Ref & \\
\hline
\end{tabular}


Stunted pupils were likely to have irregular school attendance $(\mathrm{AOR}=9.4 ; 95 \% \mathrm{CI}: 4.42-19.93 ; p<.001)$ examination $(\mathrm{AOR}=3.9 ; 95 \% \mathrm{CI}: 1.67-8.92 ; p<.01)$. and failed in recent end of year examinations ( $\mathrm{AOR}=23.6$; 95\%CI:10.24-54.19; $p<.001)$. Older age pupils were likely to have irregular school attendance (AOR=3.7; 95\% CI: $1.73-7.73 ; p<.001)$ as compared to younger pupils. And pupils who belonged in a household with inadequate food were likely to fail at the end of year

\section{DISCUSSION}

This study aimed to determine the prevalence of stunting and its associated factors among public primary school pupils in Kasulu District. We observed that $31.8 \%$ of the study population was stunted, in which $49.6 \%$ were girls and $50.4 \%$ were boys, showing both girls and boys being equally affected by stunting. Also, inadequate household food supply; reduced frequency of eating proteinous food, and poor hand hygiene after toilet use significantly were associated with stunting. Importantly, stunting negatively affected school attendance and subsequent pupil's academics. It has shown that inadequate food at home reduce children learning capacity at school.

\section{Prevalence of Stunting}

This prevalence of stunting is high and according to WHO is of public health concern requiring interventions. We observed a $6 \%$ higher prevalence than the global prevalence of stunting among under-five children which is at $26 \%,{ }^{8}$ but, was about $6 \%$ lower than the African region stunting prevalence ${ }^{9}$, and lower than that found by Galgamuwa et al. ${ }^{16}$

Other studies done in the African region among a similar population have reported varying prevalence of stunting. For instance, Biadgilign et al. ${ }^{17}$ and Derso T, et al ${ }^{11}$ found the prevalence of stunting to be $46.7 \%$ and $58.1 \%$, respectively while Bamba $I$, et al reported a prevalence of $29.4 \%$ among primary school children. ${ }^{12}$ Differences in prevalence were attributed to differences and similarities in study methodology, study age population, and the difference in study geographical areas.

Besides, this prevalence is 3\% lower than the estimated stunting prevalence among under-fives in Tanzania and $2 \%$ lower than what was found in THDS-MIS 2015$2016 .^{33}$ suggesting that stunting affects primary school pupils at the same magnitude as it affects children under five years of age.

Similarly, Masanyiwa et al in Nzega Tanzania found a prevalence of $26.1 \%$, and Semali et al found a prevalence of $47.9 \%,{ }^{13,14}$ these studies were done among underfives.

We observed that stunting affected public primary pupils as it was observed among under-five children. We found insignificant variation of stunting between Kasulu District public primary school and other parts of Tanzania. This prevalence is high and poses challenges to Kasulu District public health to achieving Sustainable Development Goal. It also has a negative implication for the nation, as stunting was associated with poor academic trajectory.

\section{Socio-Economic Factors}

In our study household wealth was found to be a significant factor associated with stunting. As the household wealth quintile increases, the risk for stunting decreases. This corroborates with findings in a study done by Makoka et al, Semali et al and Masanyiwa et al. ${ }^{18,14,13}$.

It also concurs with findings in the studies done in African countries and other parts; Herrador et $a l .{ }^{34}$, and the study done in Sri-Lanka by Galgamuwa et al. ${ }^{16}$

\section{Dietary Practices}

We found that inadequate household food security was associated with stunting. A similar observation was observed by Nyaruhucha et al, ${ }^{22}$ in Simanjiro district Tanzania reported that $87 \%$ of stunted children came from households with food insecurity of which food was described as poor in terms of both quantity and quality. ${ }^{22}$ However, food diversity and plate sharing during eating on multivariable analysis were not associated with stunting as it was found in a study done by Masanyiwa et al, ${ }^{13}$ Semali et al, ${ }^{14}$ and Chirande et al, ${ }^{19}$ the difference can be explained by the difference in the age group of study populations and study methodology

\section{Water, Sanitation, and Hygiene}

Additionally, we found that; stunting among primary pupils was associated with poor hand hygiene after visiting toilets. This habit may have predisposed them to water-borne diseases and to intestinal worm infestation and diarrhoea diseases, which if left untreated deprives nutrients and chronic deprivation lead to stunting. Similar results were found by Masanyiwa et al, ${ }^{13}$ in Nzega, Tanzania, Biadgilign et al, ${ }^{17}$ in Ethiopia, Kofuor et $a l,{ }^{25}$ in Ghana.

\section{Academic Performance /Attendance}

We observed that majority of stunted children had high odds of performing poorly in the recent overall end of year examinations. Similar observations were found among school children in Malaysia, ${ }^{21}$ in North Tripura, ${ }^{22}$ Ethiopia, ${ }^{18}$ and Burkina Faso. ${ }^{23}$ The impact of stunting on academic performance has negative onward academic trajectories and well-being. We further observed that; older and stunted pupils had irregular school attendance; however, pupil's age was not associated with stunting. These findings were like findings found by SR NAIK et al and Stuber et al. ${ }^{24,25}$

\section{Study Limitations}

Some study participants did not understand Kiswahili which necessitated finding interpretation which may have resulted in misclassification biases. Data collection was done in the rainy season where vegetables and fruits were readily available which may have shifted the study results to either direction, and about $30 \%$ of study respondents were in class one who had not experienced school life hence not exposed to starvation for a long time during class hours which may have affected stunting prevalence.

Academic performance was examined for about $70 \%$ of respondents as class one pupils had no examination results, this may have affected the association

\section{CONCLUSION}

The study was able to determine the socio-economic factors which were associated with stunting. It was found 
that household wealth predetermined a pupil's stunting status. Moreover, household food insecurity and not including protein-containing food in daily meals contributed significantly to pupils stunting. Also, poor hand wash after visiting the toilet was found to be associated with stunting. More stunting has a significant association with school academic performance and attendance.

\section{Recommendations}

Primary schools based programs to address stunting such as school feeding program should be implemented and strengthened, making use of locally available foods, coupled with creating community awareness on the inclusion of food diversity in a meal, strengthening water, sanitation, and hygiene in schools, and channel the community program such as Tanzania Social Action Funds (TASAF) to the household with food insecurity by emphasizing on micro-agro-business to supplement household with food insecurity.

Other researches with superior methods should be done in Kasulu District to establish causality, and to find out whether the stunted pupils received or missed nutrition interventions during under-fives and how to mitigate factors associated with stunting among public primary school pupils.

\section{REFERENCES}

1. AbubakarA, Uriyol, MsuyaS, SwaiM, Stray-PedersenB. Prevalence and risk factors for poor nutritional status among children in the Kilimanjaro region of Tanzania.Int J Environ Res Public Health. 2012;9(10):3506-3518. doi:10.3390/ijerph9103506. Medline

2. United Nations International Children's Emergency Fund. Tanzania. Nutrition factsheet. 2010

3. United Nations International Children's Emergency Fund. Multisectoral Approaches to Nutrition: nutrition-specific and nutrition sensitive interventions to accelerate progress. $\mathrm{Br}$ Nutr Overv. $2013 ; 1-4$

4. FindingsM. Joint United Nations International Children'Emergency Funds - World Health Organization - The World Bank Child Malnutrition Database: Estimates for 2012 and Launch of Interactive Data Dashboards 1. 2015;2-4.

5. StatesM. Stunting Policy Brief. 2012;(9).

6. de OnisM, DeweyKG, BorghiE, OnyangoAW, BlössnerM, DaelmansB, et al. Orginal Article the World Health Organization' s global target for reducing childhood stunting by 2025: rationale and proposed actions. 2013; 9:6-26

7. BhuttaZA, AhmedT, BlackRE, CousensS, DeweyK, GiuglianiE, et al. Maternal and Child Undernutrition 3 What works? Interventions for maternal and child undernutrition and survival. 2008;371.

8. United Nations International Children's Emergency Fund, World Health Organization, The World Bank. (UNICEF-WHO- World Bank). Joint Child Malnutrition Estimates: Levels \& trends in child malnutrition.. 2012; 8-13. Internet.

9. United Nations International Children' Emergency Funds. Improving Child Nutrition, The achievable imperative for global progress.

10. de OnisM, BlössnerM, BorghiE. Prevalence and trends of stunting among pre-school children, 1990-2020. Public Health Nutr. 2012;15(1):142-148. doi:10.1017/ S1368980011001315. Medline
11. DersoT, TarikuA, BiksGA, WassieMM. Stunting, wasting and associated factors among children aged 6-24 months in Dabat health and demographic surveillance system site: A community based cross-sectional study in Ethiopia.BMC Pediatr. 2017; 17/1):96. doi:10.1 186/s12887-017-0848-2. Medline

12. Bambal, ZongoD, BagayanM, Barrettl. Successful Control of SoilTransmitted Helminthiasis in School Age Children in Burkina Faso and an Example of Community- Based Assessment via Lymphatic Filariasis Transmission Assessment Survey. 2016;1-14.

13. MasanyiwaZ, Lwelamiral. Prevalence and Factors Associated with Child Malnutrition in Nzega District, Rural Tanzania. 2016;(March).

14. SemalilA, Tengia-KessyA, MmbagaEJ, LeynaG. Prevalence and determinants of stunting in under-five children in central Tanzania: remaining threats to achieving Millennium Development Goal 4.BMC Public Health. 2015;15(1):1153. doi:10.1186/ s1 2889-0 15-2507-6. Medline

15. SurveyH, Surveyl. Tanzania. 2015;

16. GalgamuwaLS, IddawelaD, DharmaratneSD, GalgamuwaGLS. Nutritional status and correlated socio-economic factors among preschool and school children in plantation communities, Sri Lanka.BMC Public Health. 2017;17111:377. doi:10.1186/ s1 2889-017-431 1-y. Medline

17. BiadgilignS, ShumetieA, YesigatH. Does economic growth reduce childhood undernutrition in Ethiopia?PLoS One. 2016;1181:e0160050. doi:10.1371/journal. pone.0160050. Medine

18. MakokaD, MasiboPK. Is there a threshold level of maternal education sufficient to reduce child undernutrition? Evidence from Malawi, Tanzania and Zimbabwe.BMC Pediatr. 2015;15(1):96. doi: 10.1186/s12887-015-0406-8. Medline

19. Chirandel, CharweD, MbwanaH, et al. Determinants of stunting and severe stunting among under-fives in Tanzania: evidence from the 2010 cross-sectional household survey.BMC Pediatr. 2015;15(1):165. doi:10.1186/s12887-015-0482-9. Medline

20. BealT, MassiotE, ArsenaulttE, SmithMR, HiimansRJ. Global trends in dietary micronutrient supplies and estimated prevalence of inadequate intakes.PLoS One. 2017;12(4): e0175554. doi: 10.1371/journal.pone.0175554. Medline

21. Garrettl, RuelMT. Are Determinants of Rural and Urban Food Security and Nutritional Status Different? Some Insights from Mozambique. World Dev. 1999;271111:1955-1975. doi:10.1016/S0305-750X(99)00091-1. Accessed June 10, 2017. Internet.

22. NyaruhuchaCNM, MsuyaJM, MamiroPS, KerengiAJ. Nutritional status and feeding practices of under-five children in Simaniiro District, Tanzania.Tanzan Health Res Bull. 2006;8(3):162-167 Medline.

23. NgureFM, ReidBM, HumphreyJH, MbuyaMN, PeltoG, StoltzfusRJ. Water, sanitation, and hygiene (WASH), environmental enteropathy, nutrition, and early child development: making the links. 2014;1308:118-28.

24. Water, Sanitation and Hygiene. 2017;1-2

25. KofuorE, DartehM, AcquahE, Kumi-kyeremeA. Correlates of stunting among children in Ghana. 2014;

26. SarmaMSG, WijesingheDGNG, SivananthawerlT. The Effects of Nutritional Status on Educational Performance of Primary School Children in the Plantation Sector in Nuwara Eliya Educational Zone.Tropical Agricultural Research. 2015;24(3):203-214. doi: $10.4038 /$ tar.v24i3.8005.

27. YyL, AbdulW, Wm M. Mutritional status, academic performance 
and parental feeding practices of primary school children in a rural district in Kelantan, Malaysia. 2014;4(1): 144-52.

28. GhoshS, SahaH. The Role of Adequate Nutrition on Academic Performance of College Students in North Tripura. $2013 ; 3(3): 41-53$.

29. SR NAIK SI and MP. Relationship between nutrition status, intelligence and academic performance of Lambani school children of Bellary district, Karnataka. 2015;5(3):259-67.

30. StuberN. Nutrition and Students' Academic Performance How does nutrition influence students' academic. 2014;65 1-280-27(Wilder Research): 1-10.

31. The United Republic of Tanzania Kigoma Region Socio-Economic Profile Joint20 18:

32. AreasA. 2012 Population and Housing Census Population Distribution by. 2013 ;

33. Ministry of Health, Community Development, Gender Elderly and Children; Demographic Health Survey and Malaria Indictors Survey 2015-16: 2016 .

34. HerradorZ, SordoL, GadisaE, MorenoJ, Nietol, CustodioE, et al. Cross-Sectional Study of Malnutrition and Associated Factors among School Aged Children in Rural and Urban Settings of Fogera and Libo Kemkem Districts, Ethiopia. 2014;9(9): 1-11.

35. ErismannS, KnoblauchAM, DiagbougaS, et al. Prevalence and risk factors of undernutrition among schoolchildren in the Plateau Central and Centre-Ouest regions of Burkina Faso.Infect Dis Poverty. 2017;6(1):17. doi:10.1186/s40249-016-0230-x. Medline

\section{Peer Reviewed}

Acknowledgement: I would like to thank God for granting the gift of life and power to do this study, and I wish to convey my sincere appreciation to Dr Leyna Germana for her tireless supportive supervision and mentorship, without forgetting all Department of Epidemiology and Biostatics staff and Muhimbili University of Health and Allied Sciences community in general. I also appreciate Tanzania Field Epidemiology and Laboratory Training Program staffs for their financial and moral support. Invaluable appreciation goes to Kasulu District Executive Director and his staff in the department of Primary Education for giving me permission to conduct this study, and staff at kitema, Kibirizi and Bugaga primary schools for their critical assistance in data collection I further extend my sincere gratitude to pupils and their parents who heartfelt consented to participate in this study.

Furthermore, I would like to acknowledge the roles of my assistant investigators; Sifa Raphael and Winnie Reuben; they worked tirelessly to make data collection successful.

Importantly I would like to appreciate the support I got from my mother and my wife for their moral support and encouragement, there contribution in this work invaluable.

Competing Interests: None declared.

Funding: This survey was funded by Tanzania Field Epidemiology and Laboratory Training Program

Received: 27 Aug 2019; Accepted: 04 Nov 2020

Cite this article as Hiliza NJ, Germana L, Kasangala A, Joram F. Prevalence and Factors Associated with Stunting among Public Primary School Pupils in Kasulu District, Western Tanzania. East Afr Health Res J. 2020;4(2):172-181. https://doi.org/10.24248/ eahrj.v4i2.641

(C) Hiliza et al. This is an open-access article distributed under the terms of the Creative Commons Attribution License, which permits unrestricted use, distribution, and reproduction in any medium, provided the original author and source are properly cited. To view a copy of the license, visit http://creativecommons. org/licenses/by/4.0/. When linking to this article, please use the following permanent link: https://doi.org/10.24248/eahrj. v4i2.641 